\title{
MANAGEMENT APPROACH BASED ON ISO 11783-10
}

\author{
Slobodan JANKOVIĆ ${ }^{1}$, Miodrag IVKOVIĆ ${ }^{2}$, Vladimir ŠINIK $^{2}$, Dragan Kleut $^{3}$ \\ ${ }^{1}$ Amalgamations Group Pltd., Reg. Office: 862, Anna Salai, 600002 Madras, India. Corresponding author. E-mail: \\ sjankovic@sify.com \\ ${ }^{2}$ University of Novi Sad, Technical faculty "Mihajlo Pupin” in Zrenjanin, 23000 Zrenjanin, Đure Đakovića bb, \\ Republic of Serbia. \\ ${ }^{3}$ SCA, Computer System Department, Belgrade, Republic of Serbia
}

Accepted 15 October, 2011

\begin{abstract}
The implementation of various electronic subsystems in farm machineries is constantly expanding. Accordingly the new machines, instead of being mechanical systems, became advanced mechatronical systems which application call for a new approach in management. Possibility of using the new and advanced standard ISO 11783 , named ISOBUS, seems to be extremely powerful tool on it. Out of other aspects the standard defines the task management i.e. the management of farm resources and field activities (ISO 11783-10:2008). The paper presents the overview global farm management enabled through ISOBUS, embedded hardware and software as infrastructure for managing farm resources and in field activities and in that way efficiency improvement of overall activities at particular farm.
\end{abstract}

Keywords: Management, ISOBUS, Agriculture.

\section{INTRODUCTION}

Amongst many factors of interest in improvement of one existing management system there are two extremely important. The first one is availability of acquiring the accurate data of interest for the process which has to be managed. The second one is, definitely, the possibilities that measures which would be found as needful for management improvement (based on analyses of acquired data) can be implemented. In addition it is of great importance to reduce the time from the moment of determination what should be the new management measures which has to be implemented till the moment of lunching those measures in the process. That task is mainly oriented to the technical managers which are under great pressure to satisfy market driven dements for fast and reliable improvement of the process.

There are a lot of data which can be acquired and which are relevant for global management of agricultural business. A few of them which are of particular interest for management in agriculture. Those are data which gave insight to the:

1. Expenses in energy consumption. Since energy aspect is of especial interest nowadays, for appropriate management decisions, the energy consumption, especially fuel consumption by agricultural machineries, is of great importance.

2. Duration of each in-field operation. Actually, duration of particular operation is of the importance since it can make serious impact to the total efficiency of agricultural business. Firstly, the longer time in operation means more expenses based on expenses for employees involved in it. In addition, longer duration of particular operation can reduce possibilities that some other operation can be done on time (based on insufficient machine recourses). It is evidently that insufficiency of machines can be solved with new investment but, that is, again, related to the higher expenses.

3. Expenses related to the application of sprayers and fertilizers. Those expenses are not only direct expenses but also indirect expenses since applying of more pesticides and fertilizer creates more problems in ecology.

4. Expenses related to the crops removing. Etc.

One can easily recognize that acquiring data from agricultural operations and in that way enabling managers to make appropriate decisions is extremely difficult. For example, it is obvious that fuel consumption can be measured very difficult in the way which would enabled its allocation to each 
particular field and operation. Or, buy using sprayers and fertilizers, it is close to impossible to measure mass of chemicals disposed to each particular field and to put that in correlation with yield from that field.

\section{THEORETICAL APPROACH TO THE NEW PROTOCOLS FOR FARM MANAGEMENT}

The concept of the system defined in ISO 11783 is based on common situation on nowadays machinery already fitted with electronic networks which are in use for data interchanging among different controllers and their instruments. All those systems are covered with ISO 11898 and ISO 11783 standards. The last one is of particular interest for

- (Part 14-Sequence Control) improvement in management activities in agriculture since it contents the following parts:

- Part 1 - General Standard

- Part 2 -Physical Layer

- Part 3 - Data Link Layer

- Part 4 - Network Layer

- Part 5 - Network - Management Layer

- Part 6-Virtual Terminal

- Part 7 - Implement Messages Layer

- Part 8-Drive Train

- Part 9-Tractor ECU

- Part 10 - Task Controller \& Management - The part important for the managers (see Fig. 1)

- Part 11 - Data Dictionary

- Part 12 - Diagnostic Services

- Part 13 - File Server

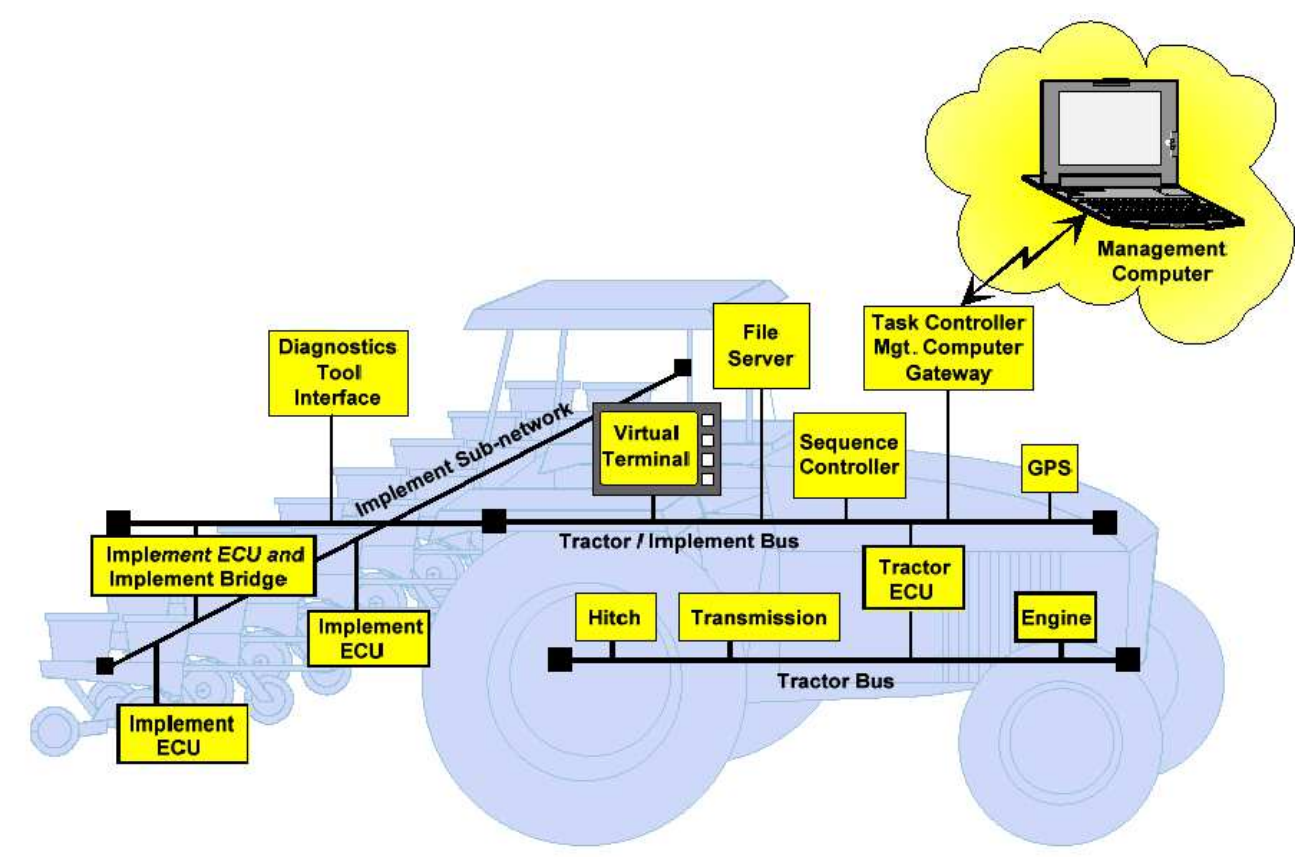

Figure 1: Hardware base for Task Control and Management on farm machineries.

In spite of the fact that there are a few protocols which are in use on agricultural machines' networks it is of importance to be noted that all of them are based, mainly, on the same physical layer which is, nowadays widely accepted by all producers. That is physical layer defined through ISO 11898 or frequently named as CAN 2.0B. As it is given in Fig. 2- right, all data which has to be transferred through this layer have to be organized in frames which start with SOF (Start of Frame) character, followed by 29 bits CAN identifier, RTR character, Control Field, 0 to 8 byte Data Field, CRC Field, Acknowledge Field and EOF (End Of Frame) bits.

From the message format is clear that data of interest for the managers would be placed in Data Field. But, to be in position to use those data a few problems have to be solved. Those problems are mainly related to the CAN identifier (ID).

As it is defined by ISO 11898 CAN ID has to be 29 bits long. But, different protocols use that identifier in different ways. For this investigation it is of importance to be oriented to the protocols which are dominant for engine and implements. As per situation in the market those controllers are mainly based on SAE J 1939 protocol. SAE J 1939 is a protocol with all 7 layers, but for the first two layers (lower two layers) it takes definition from ISO 11898 i.e. CAN 2.0B bus. Before establishing data acquisition from CAN bus it has to be analysed in which way SAE J 1939 uses CAN's identifier. In 
brief, in J 1939 the first 3 bits of ISO 11898 Identifier (ID) are in use for priority (000 for the highest priority and 1111 for the lowest priority). The next 18 bits are in use for PGN or Parameter Group Number.

The PGN is the key element for understanding possibilities for service data acquisition from the existing vehicle's network. Actually, all data which have to be sent to the CAN bus are organized in the groups. For example, all data relevant for the electrical transmission controller would be placed in the data message (according to the SAE J 1939 terminology: PDU i.e. Protocol Data Unit) in which PGN would be 61442 and 61445. Data available in messages (i.e. messages Data Fields) with stated PGN(s) would be: transmission selected gear, transmission actual gear ratio, percent of clutch slip, transmission input shaft speed, etc. It is clear that lot of data significant for transmission service load are already available on the vehicle bus in the messages with appropriate PGN's.

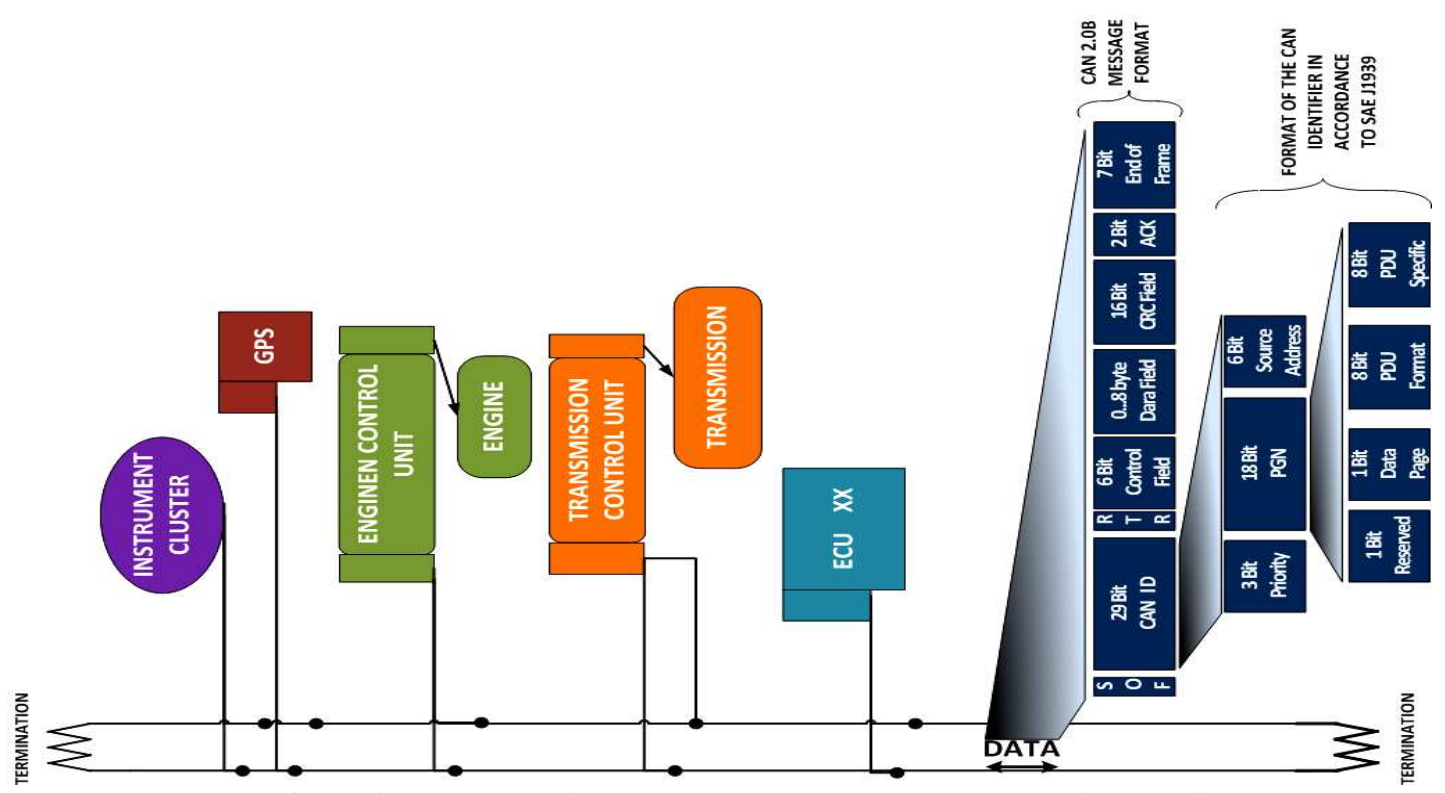

Figure 2: CAN bus - base for implementation farm management in line with ISO 11783.

It is clear that for the concept of acquiring service load data it is essential to be familiar with the PGN concept. Unfortunately, the concept of PGN and establishing CAN identifier is not as simple as said above. Strictly speaking, PGN has 4 parts: Reserved bit, Data Page bit, PDU Format -8 bits and PDU Specific -8 bits. Also, it has to be recognized that PDU Specific can be defined in two different ways based on the value of PDU Formats: as the Destination Address (for PDU Format values 0 to 239) or as Group Extension (For PDU Format values 240 to 255). All of this makes approach to the messages on the CAN bus (PDU) very complicated and can cause a lot of problems. As it was explained, defining the proper PGN is not sufficient for successful data acquisition i.e. acquiring of data of interest from the CAN bus. It is also important to know the position of the value of interest in Data Field. The Data Field is 8 bytes long and encloses different data. Their allocation in Data Field must be known for proper data extraction.

Based on explained concept of data flow trough CAN bus it is obvious that first interest in establishing the system for data acquisition has to be oriented to providing of adequate software tools for PGN, CAN Identifier (ID) and Data Field evaluation.

\section{THE MANAGEMENT ASPECT - A NEW BUSINESS MODEL}

As it was noted ISO 11783, Part 10 defines the task management, communication between task controller and electronic control units as well as data transfer between farm management information system and mobile implement control system.

The farm management information system is the complex of farm computers and management software while the mobile implement control system is group of devices that are coupled by ISO 11783 network. A task controller is the primary electronic control unit (ECU) on the agricultural machine responsible for sending, receiving and logging of process data.

The central atomic data management unit that comprises the agricultural resources, products, and operations is called task. A maximum of one task 
can be active concurrently on a single task controller.

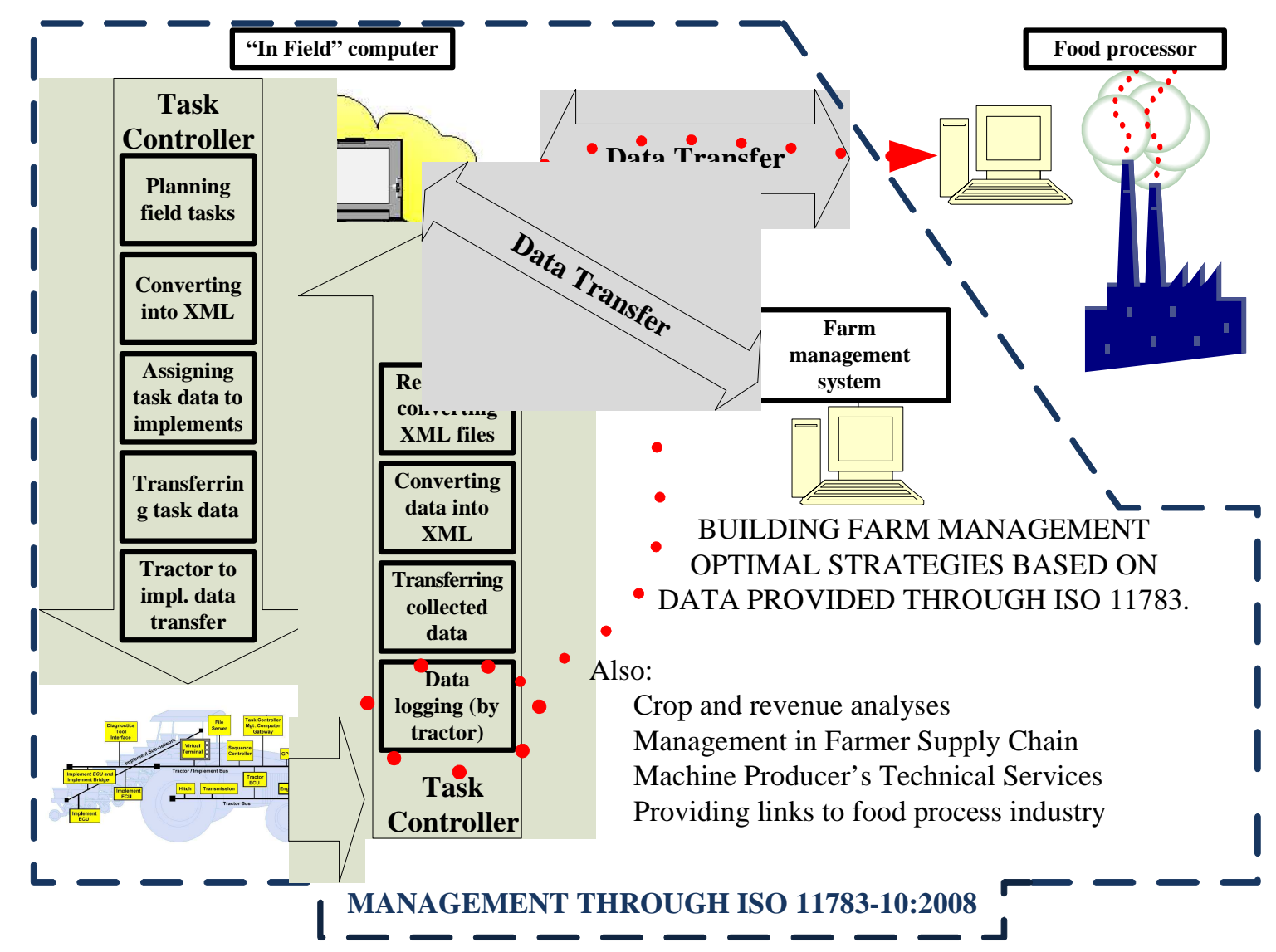

Figure 3: The farm management information system with implementation of ISO 11783-10.

As it was given in Fig. 3. the importance of Tack Controller comes from the possibility that it can be configured to control each particular task i.e. application which means that it contains all data for particular in field application. A task contains all necessary data for a field operation. The Task Controller also sends out all commands to a controlled implement during field operation. A Task Controller is typically used for example for precision farming. When the application rate of a sprayer depends on the current position the Task matter Controller will prepare the sprayer with updated data during field operation. Another important aspect of the Task Controller is the logging capability. A Task Controller will not only accept and run tasks. A Task Controller will also $\log$ and report actual field application data back to the farm management system.

\section{EXPERIMENTAL RESULTS ON APPLICATION}

This section deals with implementation of the approach explained above i.e. system based on ISO
11783. The agricultural tractor with service mass of $2850 \mathrm{~kg}$, mass distribution front : $1250 \mathrm{~kg}$, rear: 2600 $\mathrm{kg}$, rated engine power of $66 \mathrm{~kW}$ at $2250 \mathrm{rpm}$ with transmission concept $6 \times 2$ gear ratio forward, $4 \mathrm{WD}$ and with it's own CAN bus was fitted with Watch Dog (WD) hardware, Extension Module (EM) hardware and GPRS modem as given in the Fig 4. On the "receiving" side it was established the server with installed "Professional CAN Terminal" software and receiving GPRS modem.

Tractor was submitted to the two types of tests:

- Regular service application (haulage, in field operation, etc.) as per predefined scenario and

- Non regular service application i.e. special tests created for in detail investigation of CAN based acquisition system's capability.

The important issue related to the management is given in the Fig. 3. As it is given in the figure the management process starts before operation starts. Actually, the manager of the process has to define the task on farm computer in which he can allocate different field in which agricultural machineries will 
perform operations. That part is visualized with at the bottom right on Fig. 4 where one can allocate fields marked with A, B, C, etc. Once when some kind of "splitting" of whole agricultural area of interest was done one has to define parameters of the
DDE. It has to be recognized that in example given in Fig. 4 the DDE defines the deposit of the compost. It is obvious that, depending on operation, this parameter can be different.

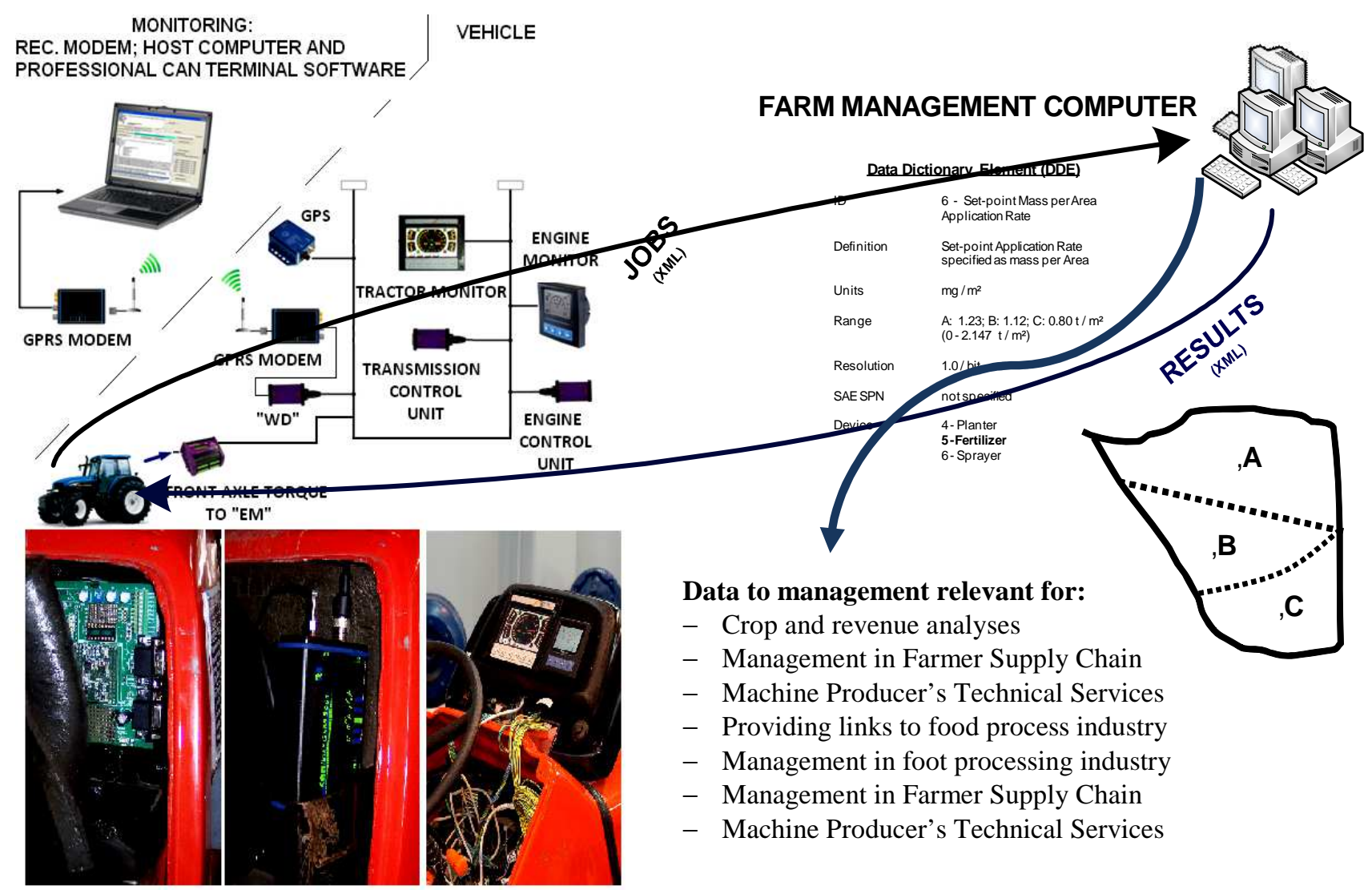

Figure 4: The farm management information system in application.

Once when parameters were defined the whole DDE has been transferred (XML format) to the machinery. Important aspect is that, in line with technology of interconnection of particular controller built in agricultural machine, controller will enable performing of the operation in the field as per defined DDE and as per request from the farm management.

At the end of operation agricultural machine establish communication with the farm computer and transfer the results of operation back to the person who manages the process.

It has to be recognized that in this way all data of interest for the management process has been transferred back. If we take in consideration that all operations, from ploughing till the removing the crop from the field are performed in the same way the set of available data would be at the level which will enable to the manager to analyze the efficacy of the strategy he implemented and to make corrections for the next season. (This aspect is given in Fig 3 left side).

Moreover, implementation of Task Controller enabled significantly new approach to the business. By taken in consideration the whole picture i.e. possibilities for shearing information provided trough Task Controller among:

\section{CONCLUSION}

The process of management in agricultural applications has pass trough significant changes which are caused by dramatically improvement in technology. The latest technologies based on standard protocols in line with ISO 11783 enables to the managers to deal with accurate data which are acquired in real time during all in field operations. In that way the manager is in position not only to have relevant information for his strategic decision, but also to implement the new concept immediately after its' design. Instead to be oriented to the indirect data 
acquiring and to deal with limited number of parameters the manager is in position to work with large set of data in real time.

The new concept is accomplished by building up dedicated hardware and software. The results of initial testing of all developed components indicate their good performances. Through initial testing of the development platform it was accomplished accurate acquiring data from agricultural tractor and its implementation in real service conditions. All conducted test were done with approach named as "Manager decisions transfer back" to the agricultural system (DDE transfer) with the results which confirmed that chosen approach is generally applicable for different agricultural systems which consider local networks.

\section{REFERENCES}

Etschberger, K. (2001). Controller Area Network. IXXAT Automation $\mathrm{GmbH}$, Weingarten, Germany.

Lawrenz, W. (2007). CAN System Engineering: From Theory to Practical Applications. Springer.

Paret, D. (2007). Multiplexed Networks for Embedded Systems. West Sussex, UK: John Wiley \& Sons, Ltd., Chichester.

Pfeiffer, O., Ayre, A., \& Keydel, Ch. (2008). Embedded Networking with CAN and CANopen. Massachusetts, USA: Copperhill Media Corporation, Greenfield.

Radojkovic, D., Sajfert, Z., Klarin, M., \& Cvijanovic, J. (2010). The influence of modified method of current observations on shortening of production cycle in medium-size companies. Technics Technologies Education Management-TTEM, 5(4), 889-895.

Voss, W. (2008). A Comprehensible Guide to J1939. Massachusetts, USA: Copperhill Media Corporation, Greenfield. 